\title{
SEISMOLOGICAL CONSTRAINTS ON CONVECTIVE PENETRATION IN THE SUN
}

\author{
G. BERTHOMIEU ${ }^{1}$, P. MOREL 1 , J. PROVOST ${ }^{1}$ \\ 1 Cassini , URA CNRS 1362, Observatoire de la Côte d'Azur, Nice \\ J.-P. ZAHN ${ }^{2,3}$ \\ ${ }^{2}$ Observatoire Midi-Pyrénées, Toulouse \\ ${ }^{3}$ Columbia University, New York
}

ABSTRACT Penetrative convection is expected below stellar convection zones, where it should achieve a nearly adiabatic stratification. A theoretical prediction of the penetration depth has been recently made by Zahn (1991) which includes an arbitrary parameter $\zeta$ depending on the properties of the convective motions. We use the helioseismological constraints to calibrate the value of this parameter.

\section{SOLAR MODELS WITH CONVECTIVE PENETRATION.}

A theoretical prediction for the depth of the convective penetration has been made recently by Zahn (1991). It is largely inspired by laboratory experiments and by recent numerical simulations of convection in a stratified medium. These show that convective penetration is due mainly to plumes which originate in the superadiabatic region and penetrate into the stable domain, which they render nearly adiabatic over some dintance. The linearization of the variation of the radiative flux, which is due mainly to the increase with depth of the radiative conductivity $\chi\left(16 \sigma T^{3} / 3 \rho \kappa\right.$ with the usual notations), leads to the following estimate for the extent of penetration $L_{p}: L_{p} / H_{P}=\zeta / \chi_{P}$, where $H_{P}$ is the pressure scale-height and $\chi_{P}=(\partial \log \chi / \partial \log P)_{\text {ad }}$.

The parameter $\zeta$ is related to the third moment of the convective velocity; it should be close to unity, but presumably a little less, since the plumes are probably less sharply

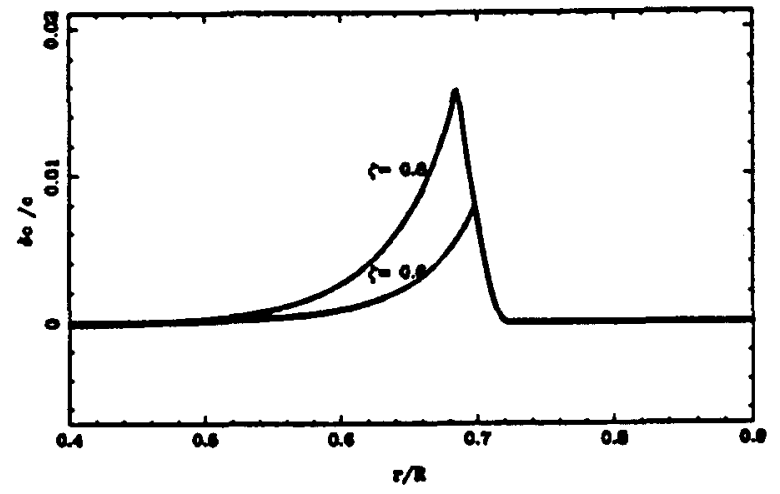

FIGURE I Variation of the difference of sound velocity between models with convective penetration and the reference solar model below the convection sone for difterent values of $\zeta$. 


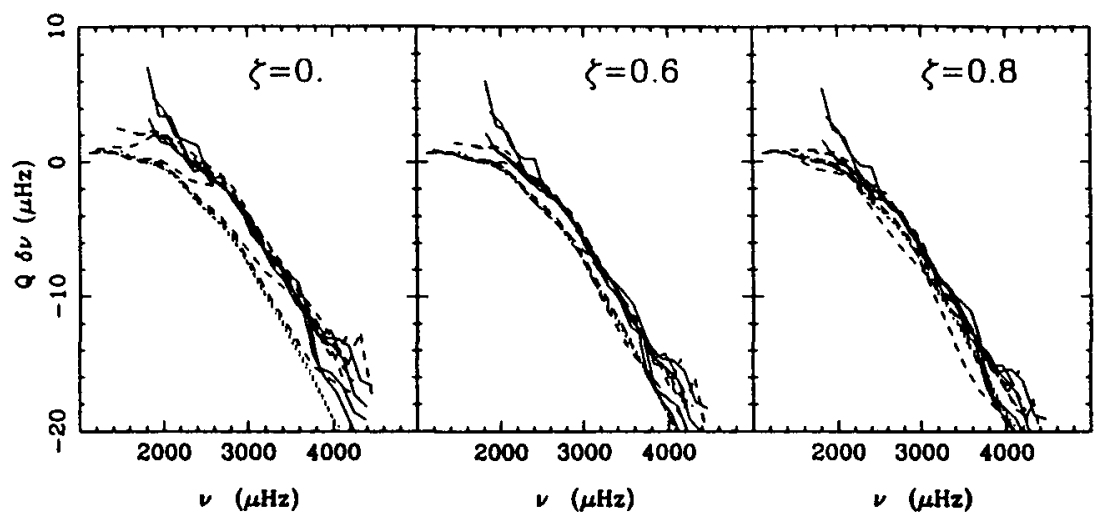

FIGURE II Relative difference between the p-mode frequencies of three models with convective penetration $\zeta=0$ (reference model), $\zeta=0.6, \zeta=0.8$ and the observed periods given by Libbrecht $e t$ al. as a function of the frequency for degrees $l=0,1,2,3,4,5$ (full line), $l=10,20,30,40,50$ (dashed line), $l=70,100$ (dotted line).

focused in the penetration layer than above. Therefore, the extent of penetration is about half a pressure scale-height in the Sun, where $\chi_{p} \approx 1.8$.

A series of solar models have been built including convective penetration at the base of the convection zone, with different values of the parameter $\zeta$. The penetration region is nearly adiabatic, and its base is very sharply defined, since the temperature gradient there changes abruptly from the adiabatic to the (lesser) radiative slope within a thermal boundary layer whose thickness if of order of $1 \mathrm{~km}$ (see fig. 2 in the review by Zahn, this meeting). Results for the sound velocity are given in figure $I$.

\section{SEISMOLOGICAL SIGNATURE OF THE CONVECTIVE PENETRATION.}

\section{A) Calibration of $\zeta$ with low and intermediate degree p-modes.}

The parameter $($ can be calibrated by comparing the acoustic frequencies of models with penetration with those observed on the Sun by Libbrecht et al. (1990). The relative differences between the frequencies of the standard model and the Sun show a significant gap between the low degree $(l<20)$ and high degree $(l>50)$ modes. It was generally attributed to an underestimate of the opacity below the convection sone, which would be filled with better determinations.

The new opacities computed by Iglesias and Rogers for the Anders Grevesse mixture are indeed larger than the Los Alamos ones in that region, and they partly fill the gap. But on closer examination, they do not suppress it, as can be seen in the first plot of figure II. The two other plots of figure II show the effect of convective penetration: it brings the low degree and high degree curves even closer together. The best agreement is achieved for a value of about $\zeta \sim 0.6$. However it is necessary to keep in mind that this value depends on the physics which is included in the models, and particularily on the opacities at the base of the convection zone. It may also depend on other physical processes which have been ignored so far, such as thermal diffusion below the convection 


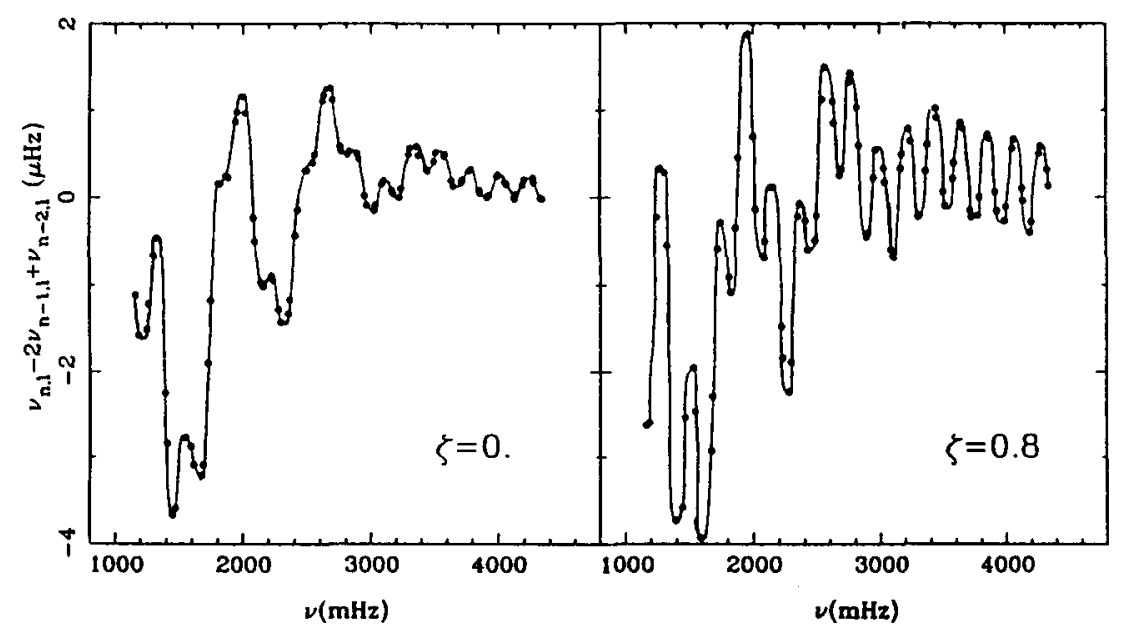

FIGURE III Variation of the differences of frequencies $\nu_{n, l}-2 \nu_{n+1, l}+\nu_{n+2, l}$ for p-modes of degree $l=0,1,2,3$ as a function of the frequency corresponding to two models: the reference model (a), and a model with $\zeta=0.8$ (b).

zone.

B) Second order differences of low degree p-modes frequencies.

To distinguish between several competing effects, on can turn to the second order difference $\Delta=\nu_{n, l}-2 \nu_{n+1, l}+\nu_{n+2, l}$, as pointed out by Gough (1990). When plotted against the frequency, this quantity has a sinusoidal behavior with two different "periods" of the order of $230 \mu \mathrm{Hz}$ and $700 \mu \mathrm{Hz}$. Theses oscillations have been interpreted by Gough: the larger "period" is due to the rapid variation of $\gamma$ in the ionisation zones, and the lower "period" is an indication for a discontinuity in the derivative of the sound velocity at the base of the convection zone.

Such a discontinuity is expected when there is convective penetration, as we have seen in figure I. Therefore it should be possible to calibrate the penetration parameter $\zeta$ by fitting the second order quantity $\Delta$ to the observations, as soon as sufficiently precise determinations of the low degree modes frequencies will become available.

\section{REFERENCES}

Anders E., Grevesse N.: 1989, Geochim. Cosmochim. Acta 53, 197

Gough D.O.: 1991, in Progress of seismology of the sun and stars, Eds Osaki Y. Shibahashi H., Springer Verlag, p 283-318

Iglesias C. A., Rogers F. J.: 1991 Astrophys. J. 371, 408-417

Libbrecht K.G., Woodard M.F., Kaufman J.M.: 1990, Astrophys. J. Supp. 74, 1129

Zahn J.P.: 1991, Astron. Astrophys. 252 179-188 